Origin of life

\section{The RNA world}

\section{from Walter Gilbert}

UNTIL recently, when one thought of the varied molecular processes at the origin of life, one imagined that the first selfreplicating systems consisted of both RNA and protein. RNA served to hold information, whereas protein molecules provided all the enzymic activities needed to make copies of RNA and to reproduce themselves. The cycle that developed a self-replicating system out of the primitive soup of amino acids and nucleotides had two radically different components ${ }^{1}$.

Now it seems possible that the informational and catalytic properties of these two components may be combined in a single molecular species. Last week in these columns Frank Westheimer ${ }^{2}$ described the discovery of enzymic activities in the RNA molecules of Escherichia coli, in which ribonuclease-P cuts phosphodiester bonds during the maturation of the transfer RNA molecule ${ }^{3,4}$, and of Tetrahymena, whose ribosomal RNA contains a selfsplicing exon ${ }^{5-7}$. If there are two enzymic activities associated with RNA, there may be more. And if there are activities among these RNA enzymes, or ribozymes, that can catalyse the synthesis of a new RNA molecule from precursors and an RNA template, then there is no need for protein enzymes at the beginning of evolution. One can contemplate an RNA world, containing only RNA molecules that serve to catalyse the synthesis of themselves".

The self-splicing intron is an RNA element that can splice itself out of an RNA molecule. This reaction should be reversible, and the intron could splice itself back into an appropriate nucleotide sequence. Thus, in the RNA world, such introns could both remove and insert themselves into the background of replicating RNA molecules. The significance of this is not the simple insertion and removal of introns, but the fact that two introns, separated from each other by another RNA element, an exon, can combine with each other so as to remove as a unit both themselves and the intervening exon from one RNA molecule and to insert into another. Thus, self-inserting introns can create transposons to move exons around. This property provides RNA with a major evolutionary facility that it otherwise lacks recombination, the ability to produce new combinations of genes. Of course the selfreplicating molecules would in any case have evolved slowly by miscopying, that is, by mutation. But transposons provide the equivalent of sex - the infectious transmission of genetic elements from one organism to another. Recombination and sex are powerful devices to permit a more useful exon to pass from one replicating structure to an unrelated one.

This picture of the RNA world is one of replicating molecules that reassort exons by transposable elements created by introns. This process builds and remakes RNA molecules by chunks and also permits the useful distinction between information and function. Information storage needs to be one-dimensional, for ease of copying, but molecules with enzymic functions tend to be tight threedimensional structures, whose forms are unrelated to the demands of any copying mechanism. (This dichotomy is most obvious today between the linear order along DNA and the structure of proteins.) In the RNA world, the structure that would be replicated has the full complement of introns. Some of the daughters, by splicing out all their introns, would convert to functional molecules, the ribozymes. A remnant of this process may be the structure of transfer RNA, where a compact secondary structure is broken up by the insertion of an intron.

The first stage of evolution proceeds, then, by RNA molecules performing the catalytic activities necessary to assemble themselves from a nucleotide soup. The RNA molecules evolve in self-replicating patterns, using recombination and mutation to explore new functions and to adapt to new niches. By using RNA cofactors, such as nicotinamide adenine dinucleotide and flavin mononucleotide they then develop an entire range of enzymic activities ${ }^{9}$. At the next stage, RNA molecules began to synthesize proteins, first by developing RNA adapter molecules that can bind activated amino acids and then

by arranging them according to an RNA template using other RNA molecules such as the RNA core of the ribosome. This process would make the first proteins, which would simply be better enzymes than their RNA counterparts. I suggest that protein molecules do not carry out enzymic reactions of a different nature from RNA molecules but are able to perform the same reactions more effectively and rapidly, and hence will eventually dominate. These protein enzymes are encoded by RNA exons, thus they, in turn, are built up of mini-elements of structure.

Finally, DNA appeared on the scene, the ultimate holder of information copied from the genetic RNA molecules by reverse transcription. After doublestranded DNA evolved there exists a stable linear information store, errorcorrecting because of its double-stranded structure but still capable of mutation and recombination. RNA is then relegated to the intermediate role that it has today no longer the centre of the stage, displaced by DNA and the more effective protein enzymes. But a few RNA enzymic activities still exist, the two described recently ${ }^{3-7}$, and possibly others in the role of ribosomal RNA or in the splicing of eukaryotic messenger RNA. The relic of this process is the intron/exon structure of genes, left imprinted on DNA from the RNA molecules that earlier encoded proteins, a residue of the basic mechanism of RNA recombination.

\footnotetext{
1. Eigen, M., Gardiner, W., Schuster, P. \& WinklerOswatitsch, R. Sci. Am. 244, 88 (1981).

2. Westheimer, F.H. Nature 319, 534 (1986).

3. Guerrier-Takada, C., Gardiner, K., Marsh, T., Pace, N. \& Altman, S. Cell 35, 849 (1983).

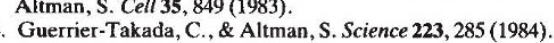

Kruger, K. et al. Cell 31, 147 (1982)

6. Cech, T.R. Int. Rev. Cytol. 93, 3 (1985).

7. Zaug, A.J. \& Cech, T.R. Science 231, 470 (1986)

8. Sharp, P.A. Cell 42, 397 (1985)

9. White, H.B. III J. molec. Evol. 7, 101 (1976).
}

\title{
Infrared detectors
}

\section{Superlattices point ahead}

\section{from Gordon C. Osbourn}

Technical developments in the past few years have made clear what the next generation of infrared detectors will be like. The need is for semiconductor materials appropriate for long-wavelength $(\geqslant 12$ $\mu \mathrm{m}$ ) imaging, at the focal planes of new telescopes and for various defence applications. Most effort has focused on the II-VI alloy system $(\mathrm{Hg}, \mathrm{Cd}) \mathrm{Te}$, but work has also begun on a recently proposed III-V system consisting of many alternating layers of thin mismatched $\operatorname{In}(\mathrm{As}, \mathrm{Sb})$ crystal layers, called strained-layer superlattices (SLSs). A crucial first step in their development has now been achieved with the successful growth of crystals of SLSs in the $\operatorname{In}(\mathrm{As}, \mathrm{Sb})$ alloy system ${ }^{1 \cdot 3}$.

The materials from which long-wavelength detectors might be made are limited by the stringency of the requirements. These imaging devices will consist of twodimensional arrays of many individual photovoltaic detector elements on a single wafer of a material which must have an energy bandgap less than or equal to $0.1 \mathrm{eV}$ at about $77 \mathrm{~K}$, the operating temperature of the infrared detector. This bandgap is smaller than that of all the III-V alloy 